\title{
A Smoothing Inexact Newton Method for Generalized Nonlinear Complementarity Problem
}

\author{
Meixia $\mathrm{Li}^{1,2}$ and Haitao $\mathrm{Che}^{2}$ \\ ${ }^{1}$ School of Mathematics and Information Science, Weifang University, Weifang, Shandong 261061, China \\ ${ }^{2}$ College of Applied Science, Beijing University of Technology, Beijing 100022, China \\ Correspondence should be addressed to Meixia Li, limeixia001@163.com
}

Received 22 November 2011; Accepted 29 January 2012

Academic Editor: Mohammad Younis

Copyright (C) 2012 M. Li and H. Che. This is an open access article distributed under the Creative Commons Attribution License, which permits unrestricted use, distribution, and reproduction in any medium, provided the original work is properly cited.

Based on the smoothing function of penalized Fischer-Burmeister NCP-function, we propose a new smoothing inexact Newton algorithm with non-monotone line search for solving the generalized nonlinear complementarity problem. We view the smoothing parameter as an independent variable. Under suitable conditions, we show that any accumulation point of the generated sequence is a solution of the generalized nonlinear complementarity problem. We also establish the local superlinear (quadratic) convergence of the proposed algorithm under the BDregular assumption. Preliminary numerical experiments indicate the feasibility and efficiency of the proposed algorithm.

\section{Introduction}

Consider the generalized nonlinear complementarity problem, denoted by GNCP $(F, G, \mathcal{K})$, which is to find a vector $x \in R^{n}$ such that

$$
F(x) \in \mathcal{K}, \quad G(x) \in \mathcal{K}^{\circ}, \quad F(x)^{\top} G(x)=0,
$$

where $F, G: R^{n} \rightarrow R^{m}$ are continuously differentiable mappings. $\mathcal{K}$ is a nonempty closed convex cone in $R^{m}$ and $\mathcal{K}^{\circ}$ denotes its polar cone.

$\operatorname{GNCP}(F, G, \mathcal{K})$ finds important applications in many fields, such as engineering and economics, and is a wide class of problems that contains the classical nonlinear complementarity problem (abbreviated as NCP); see [1-3] and references therein. To solve it, one usually reformulates it as a minimization problem over a simple set or an unconstrained optimization problem; see [4] for the case that $K$ is a general cone, and see $[3,5]$ for the case 
that $\mathcal{K}=R_{n}^{+}$. The conditions under which a stationary point of the reformulated optimization is a solution of the $\operatorname{GNCP}(F, G, \mathcal{K})$ were also provided in the literature.

In this paper, we consider the $\operatorname{GNCP}(F, G, \mathcal{K})$ for the case that $m=n$, and $\mathcal{K}$ is a polyhedral cone in $R^{n}$; that is, there exist $A \in R^{s \times n}$ and $B \in R^{t \times n}$ such that

$$
\mathcal{K}=\left\{v \in R^{n} \mid A v \geq 0, B v=0\right\} .
$$

It is easy to verify that its polar cone $\mathcal{K}^{\circ}$ has the following representation:

$$
\mathcal{K}^{\circ}=\left\{u \in R^{n} \mid u=A^{\top} \lambda+B^{\top} \mu, \lambda \in R^{s}, \lambda \geq 0, \mu \in R^{t}\right\} .
$$

In the following, the $\operatorname{GNCP}(F, G, \mathcal{K})$ is specialized over a polyhedral cone, and in the subsequent analysis, we abbreviate it as GNCP for simplicity. In [1], Andreani et al. reformulated the problem as a smooth optimization problem with simple constraints and presented the sufficient conditions under which a stationary point of the optimization problem is a solution of the concerned problem. Wang et al. [6] reformulated the problem as a system of nonlinear and nonsmooth equations and proposed a nonsmooth L-M method to solve this problem and proved that the algorithm is both globally and superlinearly convergent under mild assumptions. Zhang et al. [7] rearranged the GNCP over a polyhedral as a smoothing system of equations, then developed a smoothing Newton-type method for solving it, and proved that their method has local superlinear (quadratic) convergence under certain conditions. There are a lot of computations to decide whether the linear system is solvable or not, which is in Step 2 of the algorithm presented in [7]. The inexact approach is one way to overcome this difficulty. Inexact Newton methods have been proposed for solving NCP [8-10]. Their main idea is to solve the linear system only approximately. It seems reasonable to ask if this kind of method can be applied to the GNCP, and this actually constitutes the main motivation of this paper. In this paper, we propose a new smoothing inexact Newton algorithm with non-monotone line search for solving GNCP by using a new type of smoothing function. We view the smoothing parameter as an independent variable. The forcing parameter of inexact Newton method links the norm of residual vector to the norm of mapping at the current iterate. Under suitable conditions, we show that any accumulation point of the generated sequence is a solution of the GNCP, and we also establish the local superlinear (quadratic) convergence of the proposed algorithm under the BD-regular assumption. Some numerical examples indicate the feasibility and efficiency of the proposed algorithm.

The rest of this paper is organized as follows. In Section 2, we show some preliminaries. Stationary point and nonsingularity conditions of the GNCP are discussed in Section 3. In Section 4, we propose a kind of algorithm for solving GNCP and obtain the convergence properties of this kind of algorithm. Numerical experiments are exhibited in Section 5 and the conclusion is stated in the last section.

At the end of this section, we indicate some standard notions used in this paper. For a continuously differentiable function $F: R^{n} \rightarrow R^{m}$, we denote the Jacobian of $F$ at $x \in R^{n}$ by $F^{\prime}(x) \in R^{m \times n}$, whereas the transposed Jacobian is denoted by $\nabla F(x)$. In particular, if $m=1$, $\nabla F(x)$ is a column vector. We use $x^{\top} y$ to denote the inner product of vectors $x, y \in R^{n}$, and use $[x]_{i}$ or $x_{i}$ to denote the $i$ th component of the vector $x \in R^{n}$. The null space of a matrix $B$ is denoted by $\mathcal{N}(B)$. 


\section{Preliminaries}

In this section, we first review some definitions and basic results.

Definition 2.1. A matrix $M \in R^{n \times n}$ is said to be a $P_{0}$-matrix if every principle minor of $M$ is nonnegative.

Definition 2.2. A function $F: R^{n} \rightarrow R^{n}$ is said to be a $P_{0}$-function if for all $x, y \in R^{n}$ with $x \neq y$, there exists an index $i_{0} \in\{1,2, \ldots, n\}$ such that

$$
x_{i_{0}} \neq y_{i_{0}}, \quad\left(x_{i_{0}}-y_{i_{0}}\right)\left[F_{i_{0}}(x)-F_{i_{0}}(y)\right] \geq 0 .
$$

For vector $a \in R^{n}$, we use $D_{a}$ for $\operatorname{diag}(a)$. For a $P_{0}$-matrix, the following conclusion holds [11].

Lemma 2.3. If $M \in R^{n \times n}$ is a $P_{0}$-matrix, then every matrix of the form

$$
D_{a}+D_{b} M
$$

is nonsingular for all positive definite diagonal matrices $D_{a}, D_{b} \in R^{n \times n}$.

Definition 2.4. Suppose that $G: R^{n} \rightarrow R^{m}$ is locally Lipschitz function. $G$ is said to be semismooth at $x$ if $G$ is directionally differentiable at $x$ and

$$
\lim _{V \in \partial G\left(x+t h^{\prime}\right), h^{\prime} \rightarrow h, t \rightarrow 0^{+}}\left\{V h^{\prime}\right\}
$$

exist for any $h \in R^{n}$, where $\partial G(x)$ denotes the generalized derivative in [12].

Definition 2.5. Suppose that $G: R^{n} \rightarrow R^{m}$ is locally Lipschitz function. $G$ is said to be strongly semismooth at $x \in R^{n}$ if $G(x)$ is semismooth at $x$ and for any $V \in \partial G(x+h)$ and $h \rightarrow 0$, it holds that

$$
G(x+h)-G(x)-V h=\mathrm{O}\left(\|h\|^{2}\right) .
$$

The concept of semismoothness was originally introduced by Mifflin for functionals [13]. Qi and Sun extended the definition of semismooth function to vector-valued functions [14]. Convex functions, smooth functions, and piecewise linear functions are examples of semismooth functions. A function is semismooth at $x$ if and only if all its component functions are semismooth. The composition of semismooth functions is still a semismooth function.

Lemma 2.6 (see [14]). Suppose that $\varphi: R^{n} \rightarrow R^{m}$ is a locally Lipschitz function and semismooth at $x$. Then

(a) for any $V \in \partial \varphi(x+h), h \rightarrow 0$,

$$
V h-\varphi^{\prime}(x ; h)=o(\|h\|)
$$


(b) for any $h \rightarrow 0$,

$$
\varphi(x+h)-\varphi(x)-\varphi^{\prime}(x ; h)=o(\|h\|) .
$$

In [6], Wang et al. reformulated the GNCP as a system of nonlinear equations based on the following Fischer function [15] $\phi(a, b)=\sqrt{a^{2}+b^{2}}-a-b$ for $a, b \in R$. In [7], Zhang et al. established a type of smoothing reformulation of GNCP based on the following smoothing approximation function to the Fischer function $\phi(\epsilon, a, b)=\sqrt{a^{2}+b^{2}+\alpha \epsilon^{2}}-a-b$ for $a, b, \epsilon \in R$, where $\alpha>0$ is a constant.

In this paper, we use the following smoothing approximation function:

$$
\phi_{\alpha}(\epsilon, a, b)=a+b-\sqrt{a^{2}+b^{2}+2 \epsilon^{2}}+\frac{\alpha}{4}\left(a+\sqrt{a^{2}+4 \epsilon^{2}}\right)\left(b+\sqrt{b^{2}+4 \epsilon^{2}}\right)
$$

of the penalized Fischer-Burmeister NCP-function:

$$
\phi_{\alpha}(a, b)=a+b-\sqrt{a^{2}+b^{2}}+\alpha a_{+} b_{+}
$$

where $\alpha>0, a, b, \epsilon \in R$, and $t_{+}=\max \{0, t\}$. This NCP-function has turned out to have stronger theoretical properties than the widely used Fischer-Burmeister function and other NCP-functions suggested previously (see [16]). The latter term penalizes violations of the complementarity condition and plays a significant role from both a theoretical and a practical point of view.

It is easy to see that the following Lemma is true from [6].

Lemma 2.7. $x \in R^{n}$ is a solution of the GNCP if and only if there exist $\lambda \in R^{s}$ and $\mu \in R^{t}$, such that

$$
\begin{gathered}
\Phi_{\alpha}(A F(x), \lambda)=0, \\
B F(x)=0, \\
G(x)-A^{\top} \lambda-B^{\top} \mu=0,
\end{gathered}
$$

where $\Phi_{\alpha}(a, b)=\left(\phi_{\alpha}\left(a_{1}, b_{1}\right), \phi_{\alpha}\left(a_{2}, b_{2}\right), \ldots, \phi_{\alpha}\left(a_{s}, b_{s}\right)\right)^{\top}$ for $a, b \in R^{s}$.

Based on the relation between $\phi_{\alpha}(\cdot, \cdot)$ and $\phi_{\alpha}(\cdot, \cdot, \cdot)$, we can establish the following smoothing function to the GNCP.

Denote

$$
\Phi_{\alpha}(\epsilon, A F(x), \lambda)=\left(\begin{array}{c}
\phi_{\alpha}\left(\epsilon,[A F(x)]_{1},[\lambda]_{1}\right) \\
\phi_{\alpha}\left(\epsilon,[A F(x)]_{2},[\lambda]_{2}\right) \\
\vdots \\
\phi_{\alpha}\left(\epsilon,[A F(x)]_{s},[\lambda]_{s}\right)
\end{array}\right)
$$




$$
\begin{aligned}
& \psi_{\alpha}(\epsilon, x, \lambda, \mu)=\left(\begin{array}{c}
\Phi_{\alpha}(\epsilon, A F(x), \lambda) \\
B F(x) \\
G(x)-A^{\top} \lambda-B^{\top} \mu
\end{array}\right), \\
& H_{\alpha}(\epsilon, x, \lambda, \mu)=\left(\begin{array}{c}
\epsilon \\
\psi_{\alpha}(\epsilon, x, \lambda, \mu)
\end{array}\right) .
\end{aligned}
$$

For simplicity, we let $y=(x, \lambda, \mu)$ and $z=(\epsilon, y)$ and denote

$$
f_{\alpha}(z)=\frac{1}{2}\left\|\psi_{\alpha}(z)\right\|^{2}, \quad T_{\alpha}(z)=\frac{1}{2}\left\|H_{\alpha}(z)\right\|^{2}=\frac{1}{2} \epsilon^{2}+f_{\alpha}(z) .
$$

From Lemma 2.7, we can see that $x^{*}$ is a solution of the GNCP if and only if there exist $\lambda^{*} \in R^{s}, \mu^{*} \in R^{t}$ such that $z^{*}=\left(0, x^{*}, \lambda^{*}, \mu^{*}\right)$ is a global minimizer with zero objective function value of the unconstrained optimization problem:

$$
\min _{z \in R^{1+n+s+t}} T_{\alpha}(z)
$$

By simple calculation, we can see that the following lemma is true.

Lemma 2.8. (1) The function $\Phi_{\alpha}(\epsilon, A F(x), \lambda)$ is continuously differentiable for $\epsilon>0$ and it holds that

$$
\partial \Phi_{\alpha}(\epsilon, A F(x), \lambda) \subseteq\left(c,\left[D_{1}(x)+D_{3}(x)\right] A F^{\prime}(x),\left[D_{2}(x)+D_{4}(x)\right]\right),
$$

where

$$
\begin{aligned}
& \mathrm{c}=\operatorname{vec}\left\{c_{i}: i \in N\right\}, \quad N=\{1,2, \ldots, n\}, \\
& c_{i}=-\frac{2 \epsilon}{\sqrt{[A F(x)]_{i}^{2}+[\lambda]_{i}^{2}+2 \epsilon^{2}}}+\frac{\alpha}{4}\left[\left([A F(x)]_{i}+\sqrt{[A F(x)]_{i}^{2}+4 \epsilon^{2}}\right) \frac{4 \epsilon}{\sqrt{[\lambda]_{i}^{2}+4 \epsilon^{2}}}\right. \\
& \left.+\left([\lambda]_{i}+\sqrt{[\lambda]_{i}^{2}+4 \epsilon^{2}}\right) \frac{4 \epsilon}{\sqrt{[A F(x)]_{i}^{2}+4 \epsilon^{2}}}\right], \\
& D_{1}(x)=\operatorname{diag}\left\{1-\frac{[A F(x)]_{i}}{\sqrt{[A F(x)]_{i}^{2}+[\lambda]_{i}^{2}+2 \epsilon^{2}}}: i \in N\right\},
\end{aligned}
$$




$$
\begin{aligned}
& D_{2}(x)=\operatorname{diag}\left\{1-\frac{[\lambda]_{i}}{\sqrt{[A F(x)]_{i}^{2}+[\lambda]_{i}^{2}+2 \epsilon^{2}}}: i \in N\right\}, \\
& D_{3}(x)=\operatorname{diag}\left\{\frac{\alpha}{4}\left(1+\frac{[A F(x)]_{i}}{\sqrt{[A F(x)]_{i}^{2}+4 \epsilon^{2}}}\right)\left([\lambda]_{i}+\sqrt{[\lambda]_{i}^{2}+4 \epsilon^{2}}\right): i \in N\right\}, \\
& D_{4}(x)=\operatorname{diag}\left\{\frac{\alpha}{4}\left(1+\frac{[\lambda]_{i}}{\sqrt{[\lambda]_{i}^{2}+4 \epsilon^{2}}}\right)\left([A F(x)]_{i}+\sqrt{[A F(x)]_{i}^{2}+4 \epsilon^{2}}\right): i \in N\right\} .
\end{aligned}
$$

(2) $H_{\alpha}(z)$ is semismooth on $R^{1+n+s+t}$ and is strongly semismooth on $R^{1+n+s+t}$ if $F^{\prime}(x)$ and $G^{\prime}(x)$ are both Lipschitz continuous on $R^{n}$.

(3) $T_{\alpha}(z)$ is continuously differentiable on $R^{1+n+s+t}$ with $\nabla T_{\alpha}(z)=V^{\top} H_{\alpha}(z)$ for any $V \in \partial H_{\alpha}(z)$ and $f_{\alpha}(\epsilon, y)$ is continuously differentiable with $\nabla f_{\alpha}(0, y)=V^{\top} \psi_{\alpha}(0, y)$ for any $V \in \partial \psi_{\alpha}(0, y)$.

\section{Stationary Point and Nonsingularity Conditions}

Generally, for an optimization problem, we can obtain its stationary point when we use the existing optimization methods to solve it. Now we should study how to guarantee that every stationary point of (2.12) is a solution of the GNCP. In the following, we will discuss the conditions.

Theorem 3.1. Let $z=(\epsilon, x, \lambda, \mu)$ be a stationary point of (2.12). If $\epsilon \geq 0$ and $\nabla F(x)^{-1} \nabla G(x)$ is positive definite in $N(B)$, then $x$ is a solution of the GNCP.

Proof. Define

$$
\begin{gathered}
U=\Phi_{\alpha}(\epsilon, A F(x), \lambda), \\
V=B F(x), \\
W=G(x)-A^{\top} \mathcal{\lambda}-B^{\top} \mu .
\end{gathered}
$$

Since $z=(\epsilon, x, \lambda, \mu)$ is a stationary point of (2.12), one has

$$
\nabla T_{\alpha}(z)=0
$$


From Lemma 2.8, we have

$$
\left(\begin{array}{cccc}
1 & c^{\top} & 0 & 0 \\
0 & \nabla F(x) A^{\top}\left[D_{1}(x)+D_{3}(x)\right] & \nabla F(x) B^{\top} & \nabla G(x) \\
0 & {\left[D_{2}(x)+D_{4}(x)\right]} & 0 & -A \\
0 & 0 & 0 & -B
\end{array}\right)\left(\begin{array}{c}
\epsilon \\
U \\
V \\
W
\end{array}\right)=0
$$

that is,

$$
\begin{gathered}
\epsilon+c^{\top} U=0 \\
\nabla F(x) A^{\top}\left[D_{1}(x)+D_{3}(x)\right] U+\nabla F(x) B^{\top} V+\nabla G(x) W=0, \\
\left(D_{2}(x)+D_{4}(x)\right) U-A W=0, \\
-B W=0 .
\end{gathered}
$$

From the forth equation of (3.4), we have $W \in \mathcal{N}(B)$. Premultiplying the second equation of (3.4) by $W^{\top} \nabla F(x)^{-1}$, one has

$$
W^{\top} A^{\top}\left[D_{1}(x)+D_{3}(x)\right] U+W^{\top} B^{\top} V+W^{\top} \nabla F(x)^{-1} \nabla G(x) W=0 .
$$

Combining (3.5) with the third and forth equations of (3.4), we obtain

$$
U^{\top}\left[D_{2}(x)+D_{4}(x)\right]\left[D_{1}(x)+D_{3}(x)\right] U+W^{\top} \nabla F(x)^{-1} \nabla G(x) W=0 .
$$

From Lemma 2.8, we have that $\left[D_{2}(x)+D_{4}(x)\right]\left[D_{1}(x)+D_{3}(x)\right]$ is positive definite. Since $W \in \mathcal{N}(B)$ from the forth equation of (3.4), together with the positive definiteness of $\nabla F(x)^{-1} \nabla G(x)$ in $\mathcal{N}(B)$, we have

$$
U=0, \quad W=0 .
$$

Subtituting $U=0$ into the first equation of (3.4), we have

$$
\epsilon=0 .
$$

Together the second equation of (3.4) with (3.8) and (3.7), we have

$$
\nabla F(x) B^{\top} V=0 .
$$

Since $\nabla f(x)$ is nonsingular, premultiplying (3.9) by $F(x)^{\top} \nabla F(x)^{-1}$, one has

$$
\|V\|^{2}=0
$$

Hence, $V=0$. We complete the proof. 
Theorem 3.2. Let $z=(\epsilon, x, \lambda, \mu)$ be a stationary point of (2.12), $\epsilon>0, B$ has full row rank, and $\nabla F(x) \nabla G(x)^{\top}$ is positive definite, then $V$ is nonsingular for any $V \in \partial H_{\alpha}(z)$.

Proof. By Lemma 2.8, we know that any element $V \in \partial H_{\alpha}(z)$ can be written as

$$
V^{\top}=\left(\begin{array}{cccc}
1 & c^{\top} & 0 & 0 \\
0 & \nabla F(x) A^{\top}\left[D_{1}(x)+D_{3}(x)\right] & \nabla F(x) B^{\top} & \nabla G(x) \\
0 & {\left[D_{2}(x)+D_{4}(x)\right]} & 0 & -A \\
0 & 0 & 0 & -B
\end{array}\right),
$$

where $D_{1}(x), D_{2}(x), D_{3}(x)$, and $D_{4}(x)$ are defined in Lemma 2.8 .

In order to complete the proof, we only need to prove that

$$
D=\left(\begin{array}{ccc}
\nabla F(x) A^{\top}\left[D_{1}(x)+D_{3}(x)\right] & \nabla F(x) B^{\top} & \nabla G(x) \\
D_{2}(x)+D_{4}(x) & 0 & -A \\
0 & 0 & -B
\end{array}\right)
$$

is nonsingular.

That is to say, if and only if

$$
D^{\top}=\left(\begin{array}{ccc}
{\left[D_{1}(x)+D_{3}(x)\right] A \nabla F(x)^{\top}} & D_{2}(x)+D_{4}(x) & 0 \\
B \nabla F(x)^{\top} & 0 & 0 \\
\nabla G(x)^{\top} & -A^{\top} & -B^{\top}
\end{array}\right)
$$

is nonsingular.

Suppose that

$$
\left(\begin{array}{ccc}
{\left[D_{1}(x)+D_{3}(x)\right] A \nabla F(x)^{\top}} & D_{2}(x)+D_{4}(x) & 0 \\
B \nabla F(x)^{\top} & 0 & 0 \\
\nabla G(x)^{\top} & -A^{\top} & -B^{\top}
\end{array}\right)\left(\begin{array}{c}
p \\
q \\
r
\end{array}\right)=0
$$

which means that

$$
\begin{gathered}
{\left[D_{1}(x)+D_{3}(x)\right] A \nabla F(x)^{\top} p+\left[D_{2}(x)+D_{4}(x)\right] q=0,} \\
B \nabla F(x)^{\top} p=0, \\
\nabla G(x)^{\top} p-A^{\top} q-B^{\top} r=0 .
\end{gathered}
$$

According to the first equation of (3.15), we have

$$
q=-\left[D_{2}(x)+D_{4}(x)\right]^{-1}\left[D_{1}(x)+D_{3}(x)\right] A \nabla F(x)^{\top} p .
$$


Combining (3.16) with the third equation of (3.15), one has

$$
\left\{\nabla G(x)^{\top}+A^{\top}\left[D_{2}(x)+D_{4}(x)\right]^{-1}\left[D_{1}(x)+D_{3}(x)\right] A \nabla F(x)^{\top}\right\} p-b^{\top} r=0 .
$$

Premultiplying by $p^{\top} \nabla F(x)$ and recalling the second equation of (3.15), we get

$$
p^{\top}\left\{\nabla F(x) \nabla G(x)^{\top}+\left(A \nabla F(x)^{\top}\right)^{\top}\left[D_{2}(x)+D_{4}(x)\right]^{-1}\left[D_{1}(x)+D_{3}(x)\right] A \nabla F(x)^{\top}\right\} p=0 .
$$

By using the assumption that $\nabla F(x) \nabla G(x)^{\top}$ is positive, we obtain

$$
p=0,
$$

which, combining with (3.16), gives

$$
q=0
$$

By using the assumption that $B$ has full row rank and the third equation of (3.15), we obtain

$$
r=0,
$$

which completes the proof.

\section{Algorithm and Convergence Property}

In this section, we formally present our smoothing inexact Newton-type algorithm with nonmontone line search for solving $H_{\alpha}(z)=0$ by using the smoothing penalized FB function $\phi_{\alpha}(\epsilon, a, b)$. This nonmontone line search method was used to solve the NCP problem in [17]. Furthermore, we show the local superlinear (quadratic) convergence properties of the algorithm.

\section{Algorithm 4.1.}

Step 1. Take constants $\delta, \gamma \in(0,1), \alpha>0$, and $\epsilon^{0}>0$ such that $\gamma \epsilon^{0}<1$. Choose $\sigma \in(0,1 / 2)$.

Let $\bar{\epsilon}=\left(\epsilon^{0}, 0\right) \in R_{++} \times R^{n+s+t}$ and $y^{0}=\left(x^{0}, \lambda^{0}, \mu^{0}\right) \in R^{n+s+t}$ be an arbitary point.

Let $z^{0}=\left(\epsilon^{0}, y^{0}\right), C_{0}=T_{\alpha}\left(z^{0}\right)$, and $Q_{0}=1$. Let $\eta_{\min }$ and $\eta_{\max }$ be two constants such that $0 \leq \eta_{\min }<\eta_{\max }<1$. Choose $\eta_{0} \in\left[\eta_{\min }, \eta_{\max }\right]$. Set $\rho\left(z^{0}\right)=\gamma \min \left\{1, T_{\alpha}\left(z^{0}\right)\right\}$ and $k:=0$.

Step 2. If $H_{\alpha}\left(z^{k}\right)=0$, then stop; otherwise, let $\rho_{k}=\rho\left(z^{k}\right)$.

Step 3. Compute $\Delta z^{k}=\left(\Delta \epsilon^{k}, \Delta x^{k}, \Delta \lambda^{k}, \Delta \mu^{k}\right) \in R^{1+n+s+t}$ by

$$
H_{\alpha}\left(z^{k}\right)+H_{\alpha}^{\prime}\left(z^{k}\right) \Delta z^{k}=\rho_{k} \bar{\epsilon}
$$


Step 4. Let $\xi_{k}=\delta^{m}$, where $m$ is the smallest nonnegative integer such that

$$
T_{\alpha}\left(z^{k}+\delta^{m} \Delta z^{k}\right) \leq\left[1-2 \sigma\left(1-\gamma \epsilon_{0} \delta^{m}\right)\right] C_{k}
$$

Step 5. Set $\mathrm{z}^{k+1}=z^{k}+\xi_{k} \Delta z^{k}, k=k+1$, and

$$
\rho\left(z^{k}\right)=\min \left\{\gamma, \gamma T_{\alpha}\left(z^{k}\right), \rho\left(z^{k-1}\right)\right\}
$$

Step 6. Choose $\eta_{k} \in\left[\eta_{\min }, \eta_{\max }\right]$; set

$$
Q_{k}=\eta_{k-1} Q_{k-1}+1, \quad C_{k}=\frac{\eta_{k-1} Q_{k-1} C_{k-1}+T_{\alpha}\left(z^{k}\right)}{Q_{k}} .
$$

Go to Step 2.

From Algorithm 4.1 and [17], it is easy to see that the following remark is true.

Remark 4.2. Let the sequence $\left\{C_{k}\right\}$ and $\left\{z^{k}=\left(\epsilon^{k}, x^{k}, \lambda^{k}, \mu^{k}\right)\right\}$ be generated by Algorithm 4.1.

(i) $C_{k+1} \leq C_{k}$ for any $k$.

(ii) $T_{\alpha}\left(z^{k}\right) \leq C_{k}$ for any $k$.

(iii) $\rho\left(z^{k+1}\right) \leq \rho\left(z^{k}\right)$ for any $k$.

(iv) $\epsilon^{0} \rho\left(z^{k}\right) \leq \epsilon^{k}$ for any $k$.

(v) $\epsilon^{k}>0$ and $\epsilon^{k+1} \leq \epsilon^{k}$ for any $k$.

Theorem 4.3. Suppose that $\left\{z^{k}=\left(\epsilon^{k}, x^{k}, \lambda^{k}, \mu^{k}\right)\right\}$ is a sequence generated by Algorithm 4.1, B has full row rank, and $\nabla F\left(x^{k}\right) \nabla G\left(x^{k}\right)^{\top}$ is positive definite, $\epsilon^{k}>0$. Then Algorithm 4.1 is well defined.

Proof. From Theorem 3.2, we know that $H_{\alpha}^{\prime}\left(z^{k}\right)$ is nonsingular. Hence, Step 3 is well defined at the $k$ th iteration.

In the following, we show that the Step 4 is well defined. For $\xi \in(0,1)$, we let

$$
R^{k}(\xi)=T_{\alpha}\left(z^{k}+\xi \Delta z^{k}\right)-T_{\alpha}\left(z^{k}\right)-\xi T_{\alpha}^{\prime}\left(z^{k}\right) \Delta z^{k}
$$


and then

$$
\begin{aligned}
T_{\alpha}\left(z^{k}+\xi \Delta z^{k}\right) & =R^{k}(\xi)+T_{\alpha}\left(z^{k}\right)+\xi T_{\alpha}^{\prime}\left(z^{k}\right) \Delta z^{k} \\
& =R^{k}(\xi)+T_{\alpha}\left(z^{k}\right)+2 \xi H_{\alpha}\left(z^{k}\right)^{\top} H_{\alpha}^{\prime}\left(z^{k}\right) \Delta z^{k} \\
& =R^{k}(\xi)+T_{\alpha}\left(z^{k}\right)+2 \xi H_{\alpha}\left(z^{k}\right)^{\top}\left(-H_{\alpha}\left(z^{k}\right)+\rho_{k} \bar{\epsilon}\right) \\
& =R^{k}(\xi)+T_{\alpha}\left(z^{k}\right)-2 \xi H_{\alpha}\left(z^{k}\right)^{\top} H_{\alpha}\left(z^{k}\right)+2 \xi H_{\alpha}\left(z^{k}\right)^{\top} \rho_{k} \bar{\epsilon} \\
& \leq R^{k}(\xi)+T_{\alpha}\left(z^{k}\right)-2 \xi H_{\alpha}\left(z^{k}\right)^{\top} H_{\alpha}\left(z^{k}\right)+2 \xi \rho_{k}\left\|H_{\alpha}\left(z^{k}\right)\right\| \epsilon^{0} \\
& =R^{k}(\xi)+T_{\alpha}\left(z^{k}\right)+2 \xi\left(-T_{\alpha}\left(z^{k}\right)+\epsilon^{0} \rho_{k}\left\|H_{\alpha}\left(z^{k}\right)\right\|\right) .
\end{aligned}
$$

If $T_{\alpha}\left(z^{k}\right) \leq 1$, then $\left\|H_{\alpha}\left(z^{k}\right)\right\| \leq 1$, which, together with (4.3), implies that

$$
\epsilon^{0} \rho_{k}\left\|H_{\alpha}\left(z^{k}\right)\right\| \leq \epsilon^{0} \gamma T_{\alpha}\left(z^{k}\right) .
$$

If $T_{\alpha}\left(z^{k}\right)>1$, then $\left\|H_{\alpha}\left(z^{k}\right)\right\| \leq T_{\alpha}\left(z^{k}\right)$. Combining with (4.3), we have

$$
\epsilon^{0} \rho_{k}\left\|H_{\alpha}\left(z^{k}\right)\right\| \leq \epsilon^{0} \gamma T_{\alpha}\left(z^{k}\right) .
$$

As a result, for any $k$,

$$
\epsilon^{0} \rho_{k}\left\|H_{\alpha}\left(z^{k}\right)\right\| \leq \epsilon^{0} \gamma T_{\alpha}\left(z^{k}\right)
$$

From (4.6) and (4.9), one has

$$
\begin{aligned}
T_{\alpha}\left(z^{k}+\xi \Delta z^{k}\right) & \leq R^{k}(\xi)+T_{\alpha}\left(z^{k}\right)+2 \xi\left(-T_{\alpha}\left(z^{k}\right)+\epsilon^{0} \gamma T_{\alpha}\left(z^{k}\right)\right) \\
& \leq\left|R^{k}(\xi)\right|+\left(1-2 \xi\left(1-\epsilon^{0} \gamma\right)\right) T_{\alpha}\left(z^{k}\right) .
\end{aligned}
$$

According to Lemma 2.8, we know that $T_{\alpha}(z)$ is continuously differentiable. Hence, there exists $\bar{\xi}>0$ such that for any $\xi \in(0, \bar{\xi}), 1-2 \xi\left(1-\epsilon^{0} \gamma\right)>0$ and

$$
T_{\alpha}\left(z^{k}+\xi \Delta z^{k}\right) \leq\left(1-2 \xi\left(1-\epsilon^{0} \gamma\right)\right) T_{\alpha}\left(z^{k}\right) \leq\left(1-2 \xi\left(1-\epsilon^{0} \gamma\right)\right) C_{k}
$$

which completes the proof.

Theorem 4.4. Suppose that $\left\{z^{k}=\left(\epsilon^{k}, x^{k}, \lambda^{k}, \mu^{k}\right)\right\}$ is an infinite sequence generated by Algorithm 4.1. Then the arbitrary accumulation point $\widehat{z}$ of the sequence $\left\{z^{k}\right\}$ is a stationary point of (2.12). 
Proof. (i) Suppose that $\widehat{z}=(\widehat{e}, \widehat{y})$ is an arbitrary accumulation point of $\left\{z^{k}\right\}$, then there exists an infinite subsequence $K_{1} \subseteq\{1,2, \ldots\}$ such that $\left\{z^{k}\right\}_{K_{1}} \rightarrow \widehat{z}$ as $k \in K_{1}$ and $k \rightarrow \infty$. Without loss of generality, we assume that $K_{1}=\{1,2, \ldots\}$. That is,

$$
\lim _{\mathrm{k} \rightarrow \infty} z^{k}=\widehat{z}
$$

According to Remark 4.2 (i), (iii), and (v), we obtain that the sequence $\left\{C_{k}\right\},\left\{\rho_{k}\right\}$, and $\left\{\epsilon^{k}\right\}$ are convergent. Without loss of generality, we assume that

$$
\lim _{k \rightarrow \infty} C_{k}=\widehat{C}, \quad \lim _{k \rightarrow \infty} \rho_{k}=\widehat{\rho}, \quad \lim _{k \rightarrow \infty} \epsilon^{k}=\widehat{\epsilon} .
$$

It is easy to see that $\widehat{C} \geq 0, \widehat{\rho} \geq 0$, and $\widehat{\epsilon} \geq 0$. By Remark 4.2 (i) and (ii), we know that

$$
0 \leq T_{\alpha}\left(z^{k+1}\right) \leq C_{k+1} \leq C_{k} \leq C_{0}
$$

which means that $\left\{T_{\alpha}\left(z^{k}\right)\right\}$ is bounded.

In the following, suppose that $\hat{\rho} \neq 0$. Without loss of generality, we assume that $\left\{T_{\alpha}\left(z^{k}\right)\right\}$ is convergent and denote

$$
\widehat{T}=\lim _{k \rightarrow \infty} T_{\alpha}\left(z^{k}\right)
$$

Combining (4.3) with the assumption $\hat{\rho} \neq 0$, we have $\widehat{T}>0$. Furthermore, by using (4.14) and Remark 4.2 (iv), we obtain that $\widehat{C}>0$ and $\widehat{\epsilon}>0$. Hence, we can deduce that

$$
\begin{gathered}
\widehat{T}=H_{\alpha}(\widehat{z})^{\top} H_{\alpha}(\widehat{z}), \quad \widehat{T} \leq \widehat{C}, \\
\widehat{\rho}= \begin{cases}r, & \text { if } \widehat{T}>1, \\
\gamma \widehat{T}, & \text { if } \widehat{T} \leq 1 .\end{cases}
\end{gathered}
$$

We now break up the proof of (i) into two cases.

(1) Assume that $\alpha_{k} \geq e>0$ for all $k$, where $e$ is a constant. In this case, by (4.4) and (4.2), it follows that

$$
\begin{aligned}
C_{k+1} & =\frac{\eta_{k} Q_{K} C_{k}+T_{\alpha}\left(z^{k}\right)}{Q_{k+1}} \\
& \leq \frac{\eta_{k} Q_{K} C_{k}+C_{k}-2 \sigma\left(1-\gamma \epsilon^{0}\right) \alpha_{k} C_{k}}{Q_{k+1}} \\
& =C_{k}-\frac{2 \sigma\left(1-\gamma \epsilon^{0}\right) \alpha_{k}}{Q_{k+1}} C_{k} \\
& \leq C_{k}-\frac{2 \sigma\left(1-\gamma \epsilon^{0}\right) e}{Q_{k+1}} C_{k},
\end{aligned}
$$


for any $k$, which, together with the boundness of $\left\{C_{k}\right\}$, yields

$$
\sum_{n=1}^{\infty} \frac{2 \sigma\left(1-\gamma \epsilon^{0}\right) e}{Q_{k+1}} C_{k}<\infty
$$

On the other hand, by $\eta_{\max } \in[0,1)$ and the definition of $Q_{k}$ given in (4.4), we have

$$
\begin{aligned}
Q_{k+1} & =1+\sum_{i=0}^{k} \prod_{j=0}^{i} \eta_{k-j} \\
& \leq 1+\sum_{i=0}^{k} \eta_{\max }^{i+1} \\
& \leq \sum_{i=0}^{\infty} \eta_{\max }^{i} \\
& =\frac{1}{1-\eta_{\max }},
\end{aligned}
$$

for any $k$.

As a result, we obtain that $\lim _{k \rightarrow \infty} C_{k}=0$, which is a contradiction with $\widehat{C}>0$.

(2) Assume that $\lim _{k \rightarrow \infty} \alpha_{k}=0$. The stepsize $\widehat{\alpha_{k}}=\alpha_{k} / \delta$ does not satisfy the line search condition (4.2) for any sufficiently large $k$, that is,

$$
T_{\alpha}\left(z^{k}+\widehat{\alpha_{k}} \Delta z^{k}\right)>\left[1-2 \sigma\left(1-\gamma \epsilon^{0}\right) \widehat{\alpha_{k}}\right] C_{k}
$$

holds for sufficiently large $k$. Since $T_{\alpha}\left(z^{k}\right) \leq C_{k}$, the aforementioned inequality becomes

$$
T_{\alpha}\left(z^{k}+\widehat{\alpha_{k}} \Delta z^{k}\right)-\frac{T_{\alpha}\left(z^{k}\right)}{\widehat{\alpha_{k}}}>2 \sigma\left(1-\gamma \epsilon^{0}\right) C_{k}
$$

Since $\widehat{\epsilon}>0$ and $T_{\alpha}(\cdot)$ is continuously differentiable at $\widehat{z}$, from Lemma 2.8, we have

$$
\begin{aligned}
-2 \sigma\left(1-\gamma \epsilon^{0}\right) \widehat{T} & =-2 \sigma\left(1-\gamma \epsilon^{0}\right) \widehat{C} \\
& \leq T_{\alpha}^{\prime}(\widehat{z}) \Delta \widehat{z} \\
& =2 H_{\alpha}(\widehat{z})^{\top}\left(-H_{\alpha}(\widehat{z})+\widehat{\rho} \bar{\epsilon}\right) \\
& \leq 2\left(-\widehat{T}+\epsilon^{0} \widehat{\rho}\left\|H_{\alpha}(\widehat{z})\right\|\right) \\
& \leq 2\left(-\widehat{T}+\epsilon^{0} \gamma \widehat{T}\right) \\
& =2\left(-1+\epsilon^{0} \gamma\right) \widehat{T},
\end{aligned}
$$


where the first equality holds from (4.4) (in the form of limit), the first inequality holds from (4.22) (in the form of limit); the second equality holds from (4.1) (in the form of limit), and the third inequality holds from $\hat{\rho}\left\|H_{\alpha}(\widehat{z})\right\| \leq \gamma \widehat{T}$ by using (4.17). Hence it follows from (4.23) and $\widehat{T}>0$ that $-1+\gamma \epsilon^{0} \geq-\sigma\left(1-\gamma \epsilon^{0}\right)$, which contradicts the fact that $\sigma \in(0,1 / 2)$ and $\gamma \epsilon^{0}<1$. We complete the proof.

Similar to the proof of Theorem 4.3 in [7], the following theorem holds.

Theorem 4.5. Suppose that $\left\{z^{k}=\left(\epsilon^{k}, x^{k}, \lambda^{k}, \mu^{k}\right)\right\}$ is an infinite sequence generated by Algorithm 4.1, $\hat{z}$ is the arbitrary accumulation point of the sequence $\left\{z_{k}\right\}$, and $\hat{z}$ is a BD-regular solution of $H(z)=0$. Then

(1) the point $\hat{x}$ is a solution of the GNCP;

(2) the sequence $\left\{z^{k}\right\}$ converges to $\widehat{z}$ superlinearly. In particular, if $F^{\prime}$ and $G^{\prime}$ are locally Lipschitz continuous at $\widehat{z}$, then $\left\{z^{k}\right\}$ converges to $\widehat{z} Q$-quadratically.

\section{Numerical Experiments}

In this section, we implement Algorithm 4.1 for solving some GNCPs in order to see the behavior of Algorithm 4.1. The parameters used in the algorithm are chosen as follows:

$$
\alpha=0.01, \quad \epsilon^{0}=2.25, \quad \sigma=0.29, \quad \gamma=0.02, \quad \delta=0.2 .
$$

The following problems are tested in [5-7].

Example 5.1. Consider the implicit complementarity problems with the following form: find $y \in R^{n}$ such that

$$
y-m(y) \geq 0, \quad F(y) \geq 0, \quad F(y)^{T}(y-m(y))=0,
$$

where $m_{i}: R^{n} \rightarrow R, i=1,2, \ldots, n$, and

$$
F(y)=A y+b=\left(\begin{array}{ccccccc}
2 & -1 & 0 & 0 & \cdots & 0 & 0 \\
-1 & 2 & -1 & 0 & \cdots & 0 & 0 \\
0 & -1 & 2 & -1 & \cdots & 0 & 0 \\
& \cdots & & & \cdots & & \\
0 & 0 & 0 & 0 & \cdots & 2 & -1 \\
0 & 0 & 0 & 0 & \cdots & -1 & 2
\end{array}\right) y+\left(\begin{array}{c}
1 \\
1 \\
1 \\
\vdots \\
1 \\
1
\end{array}\right)
$$

and $m(y)=\psi(A y+b)$ with $\psi: R^{n} \rightarrow R^{n}$ being twice continuously differentiable. The following choices of function $\psi$ define our test problems.

(1) $\psi_{i}(x)=-0.5-x_{i}, \quad i=1,2, \ldots, n$,

(2) $\psi_{i}(x)=-1.5 x_{i}+0.25 x_{i}^{2}, \quad i=1,2, \ldots, n$. 
Table 1: Numerical results of Example 5.1 with $n=4$.

\begin{tabular}{llccr}
\hline$\Psi$ & ST & IT & TV & CPU \\
\hline$(1)$ & (a) & 9 & $9.7977 \times 10^{-13}$ & 0.0163 \\
$(2)$ & (a) & 9 & $4.7750 \times 10^{-14}$ & 0.0182 \\
$(1)$ & (b) & 12 & $3.9079 \times 10^{-13}$ & 0.0190 \\
$(2)$ & (b) & 13 & $2.6126 \times 10^{-13}$ & 0.0191 \\
$(1)$ & (c) & 15 & $6.9153 \times 10^{-13}$ & 0.0210 \\
$(2)$ & (c) & 19 & $6.2238 \times 10^{-13}$ & 0.0224 \\
$(1)$ & (d) & 13 & $1.0872 \times 10^{-13}$ & 0.0186 \\
$(2)$ & (d) & 12 & $5.0859 \times 10^{-13}$ & 0.0189 \\
\hline
\end{tabular}

Table 2: Numerical results of Example 5.1 with $n=8$.

\begin{tabular}{lllcr}
\hline$\Psi$ & ST & IT & TV & CPU \\
\hline$(1)$ & (a) & 19 & $6.1362 \times 10^{-13}$ & 0.1261 \\
$(2)$ & (a) & 15 & $5.5411 \times 10^{-14}$ & 0.1142 \\
$(1)$ & (b) & 17 & $1.5028 \times 10^{-13}$ & 0.1902 \\
$(2)$ & (b) & 17 & $2.4160 \times 10^{-13}$ & 0.2184 \\
$(1)$ & (c) & 19 & $2.4432 \times 10^{-13}$ & 0.2018 \\
$(2)$ & (c) & 22 & $2.6420 \times 10^{-13}$ & 0.2215 \\
$(1)$ & (d) & 33 & $6.0168 \times 10^{-13}$ & 0.2861 \\
$(2)$ & (d) & 27 & $3.2360 \times 10^{-13}$ & 0.1839 \\
\hline
\end{tabular}

The numerical results are shown in Tables 1, 2, and 3 with the following four kinds of initial points:

(a) $(0,0, \ldots, 0)^{\top}$, (b) $(-0.5,-0.5, \ldots,-0.5)^{\top}$,

(c) $(-1,-1, \ldots,-1)^{\top}$, (d) $(0.5,0.5, \ldots, 0.5)^{\top}$.

In Tables 1, 2, and 3, ST denotes initial point, IT is the iterative number, TV is the final value of $\mathrm{T}$ when the algorithm terminates, and CPU denotes the computing time in the computer, respectively. For the starting point, we choose $\lambda^{0}=(0.5,0.5, \ldots, 0.5)^{\top} \in R^{n}$ and the termination criterion for the Algorithm is $\left\|T\left(z^{k}\right)\right\| \leq 10^{-6}$.

From Tables 1, 2, and 3, we can see that Algorithm 4.1 is efficient in solving this kind of problem.

In the following, we will compare Algorithm 4.1 (denoted by Inexact) with the exact Newton algorithm with nonmontone line search (denoted by Exact). Here, our test problem is the previous problem (1), that is,

$$
\psi_{i}(x)=-0.5-x_{i}, \quad i=1,2, \ldots, n
$$

The initial point is (a) or (b). The numerical results are shown in Table 4 .

From Table 4, we can see that Algorithm 4.1 is prior to the exact smoothing Newton method when the GNCP is of relatively large scale. 
Table 3: Numerical results of Example 5.1 with $n=12$.

\begin{tabular}{lllcr}
\hline$\Psi$ & ST & IT & TV & CPU \\
\hline$(1)$ & (a) & 24 & $2.7819 \times 10^{-13}$ & 0.1453 \\
$(2)$ & (a) & 22 & $7.7064 \times 10^{-14}$ & 0.1962 \\
$(1)$ & (b) & 18 & $2.0643 \times 10^{-13}$ & 0.2003 \\
$(2)$ & (b) & 19 & $2.6558 \times 10^{-13}$ & 0.2216 \\
$(1)$ & (c) & 21 & $2.9380 \times 10^{-13}$ & 0.2421 \\
$(2)$ & (c) & 23 & $3.5009 \times 10^{-13}$ & 0.2432 \\
$(1)$ & (d) & 45 & $9.6621 \times 10^{-13}$ & 0.3056 \\
$(2)$ & (d) & 32 & $7.5216 \times 10^{-13}$ & 0.2343 \\
\hline
\end{tabular}

Table 4: Comparison of numerical results with $n=800$.

\begin{tabular}{lcccc}
\hline ST & Algorithm & IT & TV & CPU \\
\hline (a) & Inexact & 36 & $5.4366 \times 10^{-13}$ & 5.4218 \\
(a) & Exact & 87 & $9.6221 \times 10^{-13}$ & 21.3326 \\
(b) & Inexact & 31 & $2.6359 \times 10^{-13}$ & 4.1453 \\
(b) & Exact & 79 & $6.4806 \times 10^{-13}$ & 19.1962 \\
\hline
\end{tabular}

\section{Conclusion}

In this paper, we combine the smoothing function of penalized Fischer-Burmeister NCPfunction with nonmontone line search in [17] to present a new smoothing algorithm to solve the generalized nonlinear complementarity problem. We obtain that the iteration sequence generated by Algorithm 4.1 converges to a solution of the generalized nonlinear complementarity problem locally superlinear (quadratic). Preliminary numerical results show the efficiency of the algorithm.

\section{Acknowledgments}

The authors are gratefully indebted to the anonymous referees for their valuable suggestions and remarks that allowed them to essentially improve the original presentation of the paper. This paper is supported by the National Nature Science Foundation of China (Grant nos. 10901096 and 10971118) and Shandong Provincial Natural Science Foundation, China (Grant no. ZR2009AL019) and the Project of Shandong Province Higher Educational Science and Technology Program (Grant no. J09LA53).

\section{References}

[1] R. Andreani, A. Friedlander, and S. A. Santos, "On the resolution of the generalized nonlinear complementarity problem," SIAM Journal on Optimization, vol. 12, no. 2, pp. 303-321, 2001.

[2] F. Facchinei and J. S. Pang, Finite-Dimensional Variational Inequalities and Complementarity Problems, Springer, NewYork, NY, USA, 2003.

[3] C. Kanzow and M. Fukushima, "Equivalence of the generalized complementarity problem to differentiable unconstrained minimization," Journal of Optimization Theory and Applications, vol. 90, no. 3, pp. 581-603, 1996. 
[4] P. Tseng, N. Yamashita, and M. Fukushima, "Equivalence of complementarity problems to differentiable minimization: a unified approach," SIAM Journal on Optimization, vol. 6, no. 2, pp. 446-460, 1996.

[5] H. Jiang, M. Fukushima, L. Qi, and D. Sun, "A trust region method for solving generalized complementarity problems," SIAM Journal on Optimization, vol. 8, no. 1, pp. 140-157, 1998.

[6] Y. Wang, F. Ma, and J. Zhang, "A nonsmooth L-M method for solving the generalized nonlinear complementarity problem over a polyhedral cone," Applied Mathematics and Optimization, vol. 52, no. 1, pp. 73-92, 2005.

[7] X. Zhang, H. Jiang, and Y. Wang, "A smoothing Newton-type method for generalized nonlinear complementarity problem," Journal of Computational and Applied Mathematics, vol. 212, no. 1, pp. 75-85, 2008.

[8] K. Natasa and R. Sanja, "Globally convergent Jacobian smoothing inexact Newton methods for NCP," Computational Optimization and Applications, vol. 41, no. 2, pp. 243-261, 2008.

[9] B. Chen and C. Ma, "A new smoothing Broyden-like method for solving nonlinear complementarity problem with a $P_{0}$ function," Journal of Global Optimization, vol. 5, no. 3, pp. 473-495, 2011.

[10] S. Rui and C. Xu, "A smoothing inexact Newton method for nonlinear complementarity problems," Journal of Computational and Applied Mathematics, vol. 233, no. 9, pp. 2332-2338, 2010.

[11] T. Luca, F. Facchinei, and C. Kanzow, "A semismooth equation approach to the solution of nonlinear complementarity problems," Mathematical Programming, vol. 75, no. 3, pp. 407-439, 1996.

[12] F. Clarke, Optimization and Nonsmooth Analysis, John Wiley \& Sons, New York, NY, USA, 1983.

[13] R. Mifflin, "Semismooth and semiconvex functions in constrained optimization," SIAM Journal on Control and Optimization, vol. 15, no. 6, pp. 959-972, 1977.

[14] L. Qi and J. Sun, "A nonsmooth version of Newton's method," Mathematical Programming, vol. 58, no. 3, pp. 353-367, 1993.

[15] A. Fischer, "A special Newton-type optimization method," Optimization, vol. 24, no. 3-4, pp. 269-284, 1992.

[16] B. Chen, X. Chen, and C. Kanzow, "A penalized fischer-burmeister NCP-function," Mathematical Programming, vol. 88, no. 1, pp. 211-216, 2000.

[17] T. Ni and P. Wang, "A smoothing-type algorithm for solving nonlinear complementarity problems with a non-monotone line search," Applied Mathematics and Computation, vol. 216, no. 7, pp. 22072214, 2010. 


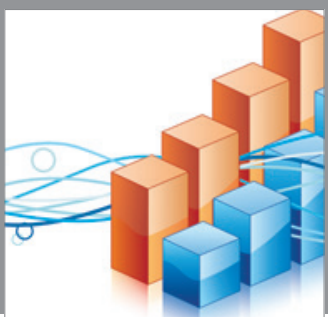

Advances in

Operations Research

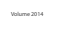

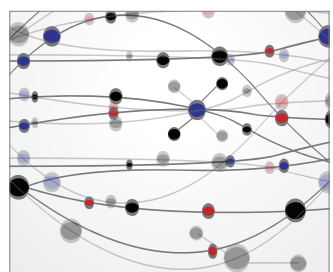

\section{The Scientific} World Journal
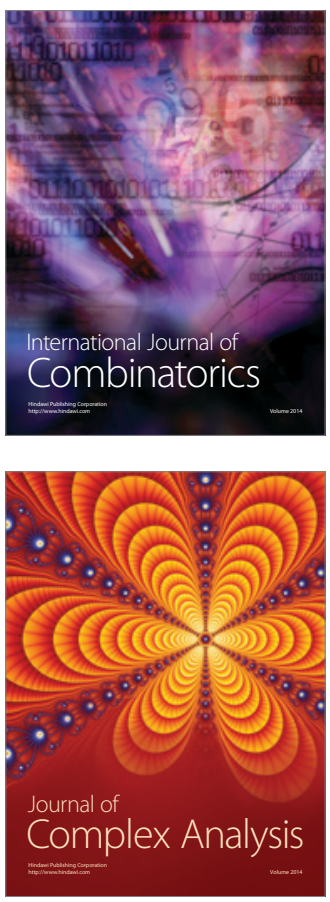

International Journal of

Mathematics and

Mathematical

Sciences
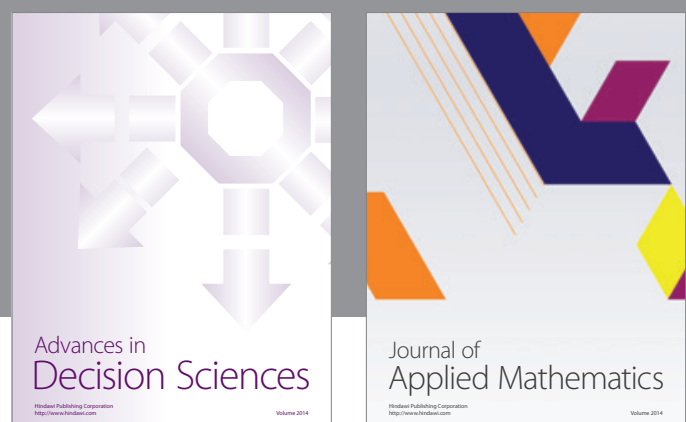

Journal of

Applied Mathematics
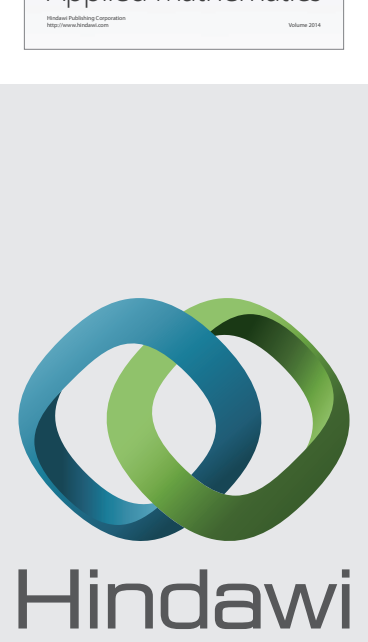

Submit your manuscripts at http://www.hindawi.com
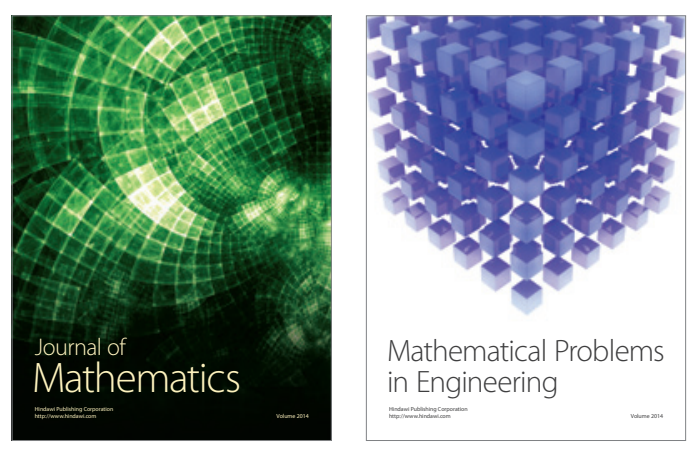

Mathematical Problems in Engineering
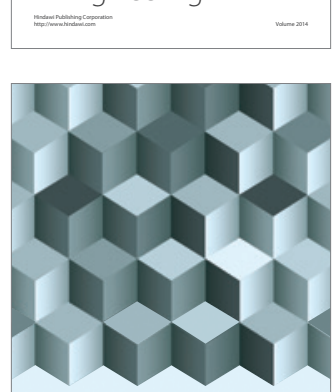

Journal of

Function Spaces
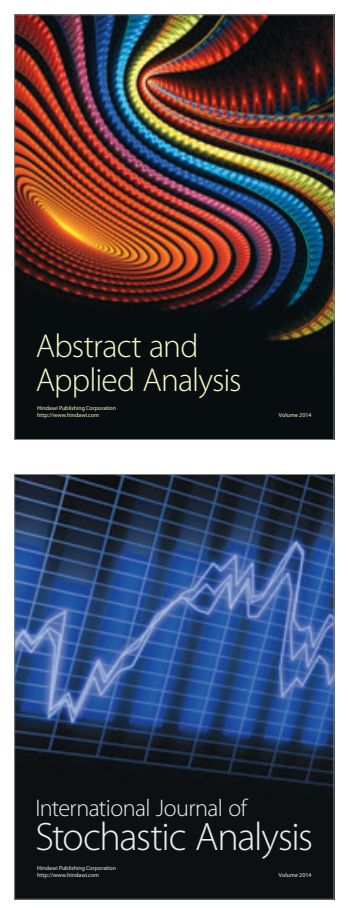

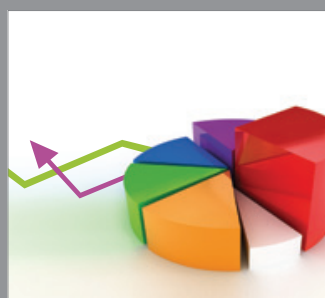

ournal of

Probability and Statistics

Promensencen
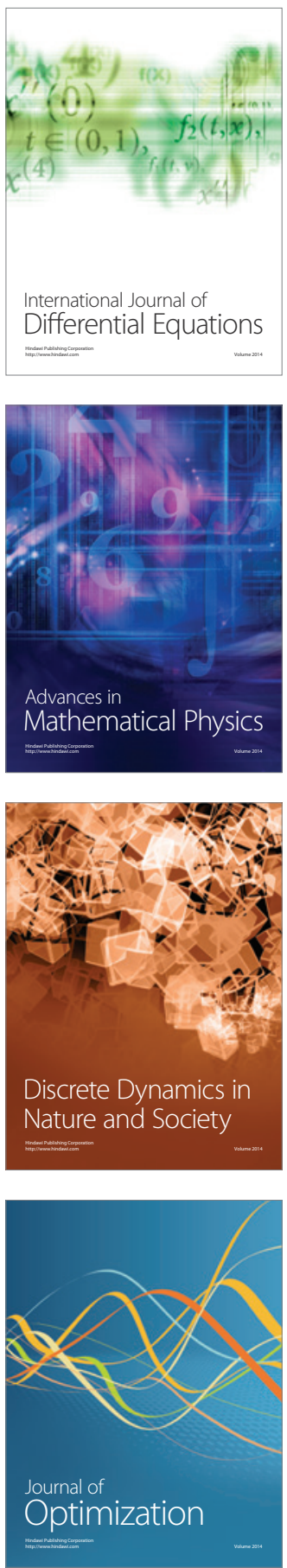